
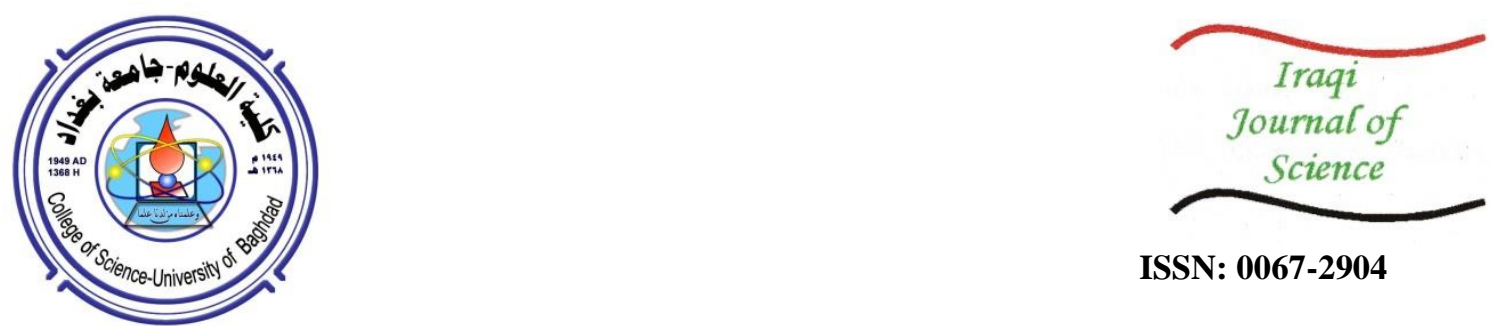

ISSN: 0067-2904

\title{
On New Types of Weakly Neutrosophic Crisp Open Mappings
}

\author{
Ali Hussein Mahmood Al-Obaidi ${ }^{\text {* }}$, Qays Hatem Imran ${ }^{2}$ \\ ${ }^{1}$ Department of Mathematics, College of Education for Pure Science, University of Babylon, Hillah, Iraq \\ ${ }^{2}$ Department of Mathematics, College of Education for Pure Science, Al-Muthanna University, Samawah, Iraq
}

Received: 18/4/2020 Accepted: 4/11/2020

\begin{abstract}
This work employs the conceptions of neutrosophic crisp $\alpha$-open and semi- $\alpha$ open sets to distinguish some novel forms of weakly neutrosophic crisp open mappings; for instance, neutrosophic crisp $\alpha$-open mappings, neutrosophic crisp $\alpha^{*}$ open mappings, neutrosophic crisp $\alpha^{* *}$-open mappings, neutrosophic crisp semi- $\alpha$ open mappings, neutrosophic crisp semi- $\alpha^{*}$-open mappings, and neutrosophic crisp semi- $\alpha^{* *}$-open mappings. Moreover, the close connections between these forms of weakly neutrosophic crisp open mappings and the viewpoints of neutrosophic crisp open mappings are explained. Additionally, various theorems and related features and notes are submitted.
\end{abstract}

Keywords: $\mathrm{NC} \alpha$-open sets, $\mathrm{NCS} \alpha$-open sets, $\mathrm{NC} \alpha$-open mappings, $\mathrm{NC} \alpha$-open mappings, $\mathrm{NC}^{* *}$-open mappings, $\mathrm{NCS} \alpha$-open mappings, $\mathrm{NCS} \alpha^{*}$-open mappings and $\mathrm{NCS} \alpha{ }^{*}{ }_{\text {-open }}$ mappings.

\section{حول أنواع جديدة من التطبيقات المفتوحة النتروسوفكية الهشه الضعيفة \\ علي حسين محمود العبيدي1* ، قيس حاتم عمران 2 \\ 1قسم الرياضيات، كلية التربية للعلوم الصرفة، جامعة بابل، العابل، حله، العراق.

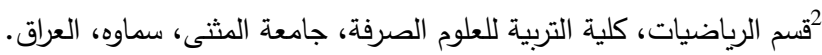

الخلاصة

هذا العمل يوظف أفكار المجموعات المفتوحة م النتروسوفكية الهشه و المفتوحة شبه م النتروسوفكية

الهثه لتميز أنواع جديدة وغير مألوفة للتطبيقات المفتوحة النتروسوفكية الهثه الضعيفة وعلى سبيل المثال

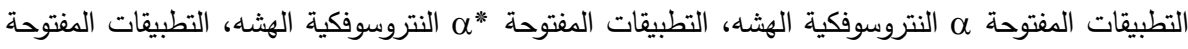

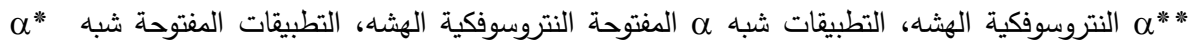

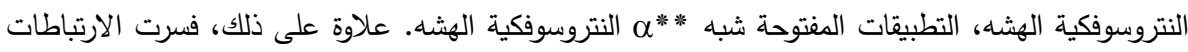

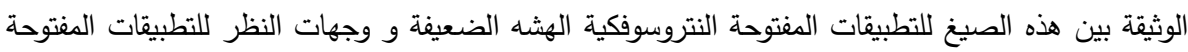

النتروسوفكية الهشه. بالإضافة الى ذلك، قدمت نظريات و خصائص و ملاحظات متنوعة.

الكلمات المفتاحية: المجموعات المفتوحة ه النتروسوفكية الهشه، المجموعات المفتوحة شبه م النتروسوفكية

الهشه، التطبيقات المفتوحة م النتروسوفكية الهشه، التطبيقات الهفتوحة *a النتروسوفكية الهشه، التطبيقات

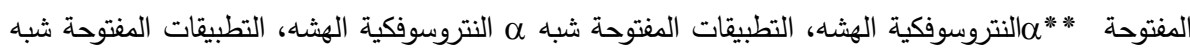

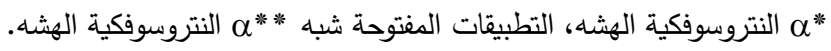

\section{Introduction}

Salama et al. [1] pioneered the abstraction of neutrosophic crisp topological space. Abdel-Basset et al. [2-7] gave a novel neutrosophic approach. Al-Hamido et al. [8] submitted the intellect of 
neutrosophic crisp semi- $\alpha$-closed sets. Banupriya et al. [9] investigated the notion of $\alpha$ gs continuity and $\alpha g$ s irresolute maps. Dhavaseelan et al. [10] exhibited the theme of neutrosophic $\alpha^{m}$-continuity. Maheswari et al. [11] introduced gb-closed sets and gb-continuity. This study aims to establish unprecedented classes of weakly neutrosophic crisp open mappings by way of examples neutrosophic crisp $\alpha$-open mappings, neutrosophic crisp $\alpha^{*}$-open mappings, neutrosophic crisp $\alpha^{* *}$-open mappings, neutrosophic crisp semi- $\alpha$-open mappings, neutrosophic crisp semi- $\alpha^{*}$-open mappings, and neutrosophic crisp semi- $\alpha^{* *}$-open mappings. Furthermore, we shall explain the close relastionships between these categories of weakly neutrosophic crisp open mappings and the conceptions of neutrosophic crisp open mappings. Furthermore, we shall show some related theorems and their features and notes.

\section{Preliminaries}

For all of this work, $(\mathcal{U}, \tau),(\mathcal{V}, \sigma)$, and $(\mathcal{W}, \rho)$ (or frugally $\mathcal{U}, \mathcal{V}$, and $\mathcal{W}$ ) always mean neutrosophic crisp topological spaces (for brevity NCTSs). For a neutrosophic crisp set $\mathcal{M}$ in a NCTS $\mathcal{U}, \mathcal{M}^{c}=\mathcal{U}-\mathcal{M}, \operatorname{NCint}(\mathcal{M})$, and $N \operatorname{Ccl}(\mathcal{M})$ signify the neutrosophic crisp complement, the neutrosophic crisp interior, and the neutrosophic crisp closure of $\mathcal{M}$, correspondingly.

Definition 2.1 [1]: For any nonempty under-consideration set $\mathcal{U}$, a neutrosophic crisp set (curtly NCS) $\mathcal{M}$ is an object holding the establishment $\mathcal{M}=\left\langle\mathcal{M}_{1}, \mathcal{M}_{2}, \mathcal{M}_{3}\right\rangle$ where $\mathcal{M}_{1}, \mathcal{M}_{2}$ and $\mathcal{M}_{3}$ are mutually disjoint sets included in $\mathcal{U}$.

Definition 2.2: Suppose that NCTS $\mathcal{U}$ contains a NCS $\mathcal{M}$, then we have the following:

1) If $\mathcal{M} \subseteq N \operatorname{Cint}(N \operatorname{Ccl}(N \operatorname{Cint}(\mathcal{M})))$, then $\mathcal{M}$ is named a neutrosophic crisp $\alpha$-open set (concisely $\mathrm{NC} \alpha-\mathrm{OS})$ [12]. The collection of all $\mathrm{NC} \alpha-\mathrm{OSs}$ of $\mathcal{U}$ is symbolized by $\mathrm{NC} \alpha \mathrm{O}(\mathcal{U})$.

2) If $\mathcal{M} \subseteq N \operatorname{Ccl}(N \operatorname{Cint}(N \operatorname{Ccl}(N \operatorname{Cint}(\mathcal{M}))))$ or, in other words, for some $\mathrm{NC} \alpha-\mathrm{OS} \mathcal{\varepsilon}$ in $\mathcal{U}$ so that $\mathcal{E} \subseteq \mathcal{M} \subseteq N \operatorname{Ccl}(\mathcal{E})$, then $\mathcal{M}$ is a neutrosophic crisp semi- $\alpha$-open set (concisely NCS $\alpha$-OS) [8]. The collection of all NCS $\alpha$-OSs of $\mathcal{U}$ is represented by $\mathrm{NCS} \alpha \mathrm{O}(\mathcal{U})$.

Remark 2.3 [8,13]: For any NCTS $\mathcal{U}$, the following claims stay valid, but not vice versa:

1) For all NC-OSs are $\mathrm{NC} \alpha$-OSs and NCS $\alpha$-OSs.

2) For all $\mathrm{NC} \alpha$-OSs are NCS $\alpha$-OSs.

Example 2.4: Let $\mathcal{U}=\{a, b, c, d\}$.

Then, $\tau_{U}=\left\{\phi_{N},\langle\{a\}, \phi, \phi\rangle,\langle\{b\}, \phi, \phi\rangle,\langle\{a, b\}, \phi, \phi\rangle,\langle\{a, b, c\}, \phi, \phi\rangle, \mathcal{U}_{N}\right\}$ is a NCTS. The class of all $\mathrm{NC} \alpha$-OSs of $\mathcal{U}$ is: $\mathrm{NC} \alpha \mathrm{O}(\mathcal{U})=\tau_{u} \cup\{\langle\{a, b, d\}, \phi, \phi\rangle\}$. The class of all NCS $\alpha$-OSs of $\mathcal{U}$ is: $\mathrm{NCS} \alpha \mathrm{O}(\mathcal{U})=\mathrm{NC} \alpha \mathrm{O}(\mathcal{U}) \cup\{\langle\{a, c\}, \phi, \phi\rangle,\langle\{a, d\}, \phi, \phi\rangle,\langle\{b, c\}, \phi, \phi\rangle,\langle\{b, d\}, \phi, \phi\rangle,\langle\{a, c, d\}, \phi, \phi\rangle$,

$\langle\{b, c, d\}, \phi, \phi\rangle\}$. The NCS $\langle\{a, b, d\}, \phi, \phi\rangle$ is a NC $\alpha$-OS (NCS $\alpha-O S)$ but not NC-OS. The NCS $\langle\{a, c, d\}, \phi, \phi\rangle$ is a NCS $\alpha$-OS but not NC $\alpha$-OS.

Proposition 2.5 [12]: For any neutrosophic crisp subset $\mathcal{M}$ of a NCTS $(\mathcal{U}, \tau), \mathcal{M} \in \mathrm{NC} \alpha \mathrm{O}(\mathcal{U})$ iff for some NC-OS $\mathcal{P}, \mathcal{P} \subseteq \mathcal{M} \subseteq N \operatorname{Cint}(\operatorname{NCcl}(\mathcal{P}))$.

Definition 2.6: Let $\ell:(\mathcal{U}, \tau) \rightarrow(\mathcal{V}, \sigma)$ be a mapping, then $\ell$ is termed:

1) Neutrosophic crisp open (briefly NC-open) [1] iff for each $\mathcal{M}$ NC-OS in $\mathcal{U}, \ell(\mathcal{M})$ is a NC-OS in $\mathcal{V}$.

2) Neutrosophic crisp $\alpha$-open (briefly NC $\alpha$-open) [14] iff for each $\mathcal{M}$ NC-OS in $\mathcal{U}, \ell(\mathcal{M})$ is a NC $\alpha$-OS in $\mathcal{V}$.

Proposition 2.7 [1]: A mapping $\ell:(\mathcal{U}, \tau) \rightarrow(\mathcal{V}, \sigma)$ is $\mathrm{NC}$-open iff $\ell(N \operatorname{Cint}(\mathcal{M})) \subseteq N \operatorname{Cint}(\ell(\mathcal{M}))$, for every $\mathcal{M} \subseteq \mathcal{U}$.

Definition 2.8 [1]: Let $\ell:(U, \tau) \rightarrow(\mathcal{V}, \sigma)$ be a mapping, then $\ell$ is called neutrosophic crisp continuous (briefly NC-continuous) iff for each $\mathcal{M}$ NC-OS in $\mathcal{V}, \ell^{-1}(\mathcal{M})$ is a NC-OS in $\mathcal{U}$.

Proposition 2.9 [1]: A mapping $\ell:(\mathcal{U}, \tau) \rightarrow(\mathcal{V}, \sigma)$ is NC-continuous iff $\ell(\operatorname{NCcl}(\mathcal{M})) \subseteq$ $\operatorname{NCcl}(\ell(\mathcal{M}))$, for every $\mathcal{M} \subseteq \mathcal{U}$.

3. Weakly Neutrosophic Crisp Open Mappings

Definition 3.1: Let $\ell:(U, \tau) \rightarrow(\mathcal{V}, \sigma)$ be a mapping, then $\ell$ is named:

1) Neutrosophic crisp $\alpha^{*}$-open (briefly $\mathrm{NC}^{*}$-open) iff for each $\mathrm{NC} \alpha-\mathrm{OS} \mathcal{M}$ in $\mathcal{U}, \ell(\mathcal{M})$ is considered a $\mathrm{NC} \alpha-\mathrm{OS}$ in $\mathcal{V}$.

2) Neutrosophic crisp $\alpha^{* *}$-open (briefly $\mathrm{NC}^{* *}$-open) iff for each $\mathrm{NC} \alpha-\mathrm{OS} \mathcal{M}$ in $\mathcal{U}, \ell(\mathcal{M})$ is considered a NC-OS in $\mathcal{V}$.

Definition 3.2: Let $\ell:(\mathcal{U}, \tau) \rightarrow(\mathcal{V}, \sigma)$ be a mapping, then $\ell$ is termed: 
1) Neutrosophic crisp semi- $\alpha$-open (briefly NCS $\alpha$-open) iff for each $\mathcal{M}$ as NC-OS in $\mathcal{U}, \ell(\mathcal{M})$ is a $\mathrm{NCS} \alpha-\mathrm{OS}$ in $\mathcal{V}$.

2) Neutrosophic crisp semi- $\alpha^{*}$-open (briefly $\mathrm{NCS}^{*}$-open) iff for each $\mathcal{M}$ as NCS $\alpha$-OS in $\mathcal{U}$, $\ell(\mathcal{M})$ is a NCS $\alpha-\mathrm{OS}$ in $\mathcal{V}$.

3) Neutrosophic crisp semi- $\alpha^{* *}$-open (briefly $\mathrm{NCS}^{* *}{ }^{*}$-open) iff for each $\mathcal{M}$ as NCS $\alpha$-OS in $\mathcal{U}$, $\ell(\mathcal{M})$ is a NC-OS in $\mathcal{V}$.

\section{Proposition 3.3}

1) Every NC-open mapping is a NC $\alpha$-open, so it is NCS $\alpha$-open, but the reverse is not valid in general.

2) Every $\mathrm{NC} \alpha$-open mapping is a NCS $\alpha$-open, but the reverse is not valid in general.

\section{Proof}

1) Let $\ell:(\mathcal{U}, \tau) \rightarrow(\mathcal{V}, \sigma)$ be a NC-open mapping and a NC-OS $\mathcal{M}$ be in $\mathcal{U}$. Then $\ell(\mathcal{M})$ is considered a NC-OS in $\mathcal{V}$. Because any NC-OS is NC $\alpha$-OS (NCS $\alpha-\mathrm{OS}), \ell(\mathcal{M})$ is considered a NC $\alpha-$ OS (NCS $\alpha$-OS) set in $\mathcal{V}$. Hence, $\ell$ is a NC $\alpha$-open (NCS $\alpha$-open) mapping.

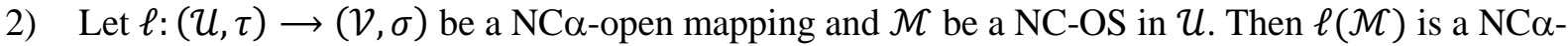
OS in $\mathcal{V}$. Because any NC $\alpha$-OS is NCS $\alpha-\mathrm{OS}, \ell(\mathcal{M})$ considers NCS $\alpha-\mathrm{OS}$ in $\mathcal{V}$. Hence $\ell$ is a NCS $\alpha-$ open mapping.

Example 3.4: Let $\mathcal{U}=\{a, b, c, d\}$.

Then, $\tau_{U}=\left\{\phi_{N},\langle\{a\}, \phi, \phi\rangle,\langle\{b\}, \phi, \phi\rangle,\langle\{a, b\}, \phi, \phi\rangle,\langle\{a, b, c\}, \phi, \phi\rangle, \mathcal{U}_{N}\right\}$ is a NCTS. The class of all $\mathrm{NC} \alpha$-OSs of $\mathcal{U}$ is: $\mathrm{NC} \alpha \mathrm{O}(\mathcal{U})=\tau_{u} \cup\{\langle\{a, b, d\}, \phi, \phi\rangle\}$. The class of all NCS $\alpha$-OSs of $\mathcal{U}$ is: $\mathrm{NCS} \alpha \mathrm{O}(\mathcal{U})=\mathrm{NC} \alpha \mathrm{O}(\mathcal{U}) \cup\{\langle\{a, c\}, \phi, \phi\rangle,\langle\{a, d\}, \phi, \phi\rangle,\langle\{b, c\}, \phi, \phi\rangle,\langle\{b, d\}, \phi, \phi\rangle,\langle\{a, c, d\}, \phi, \phi\rangle$, $\langle\{b, c, d\}, \phi, \phi\rangle\}$.

We define a mapping $\ell: \mathcal{U} \rightarrow \mathcal{U}$ by $\ell(\langle\{a\}, \phi, \phi\rangle)=\langle\{a\}, \phi, \phi\rangle, \ell(\langle\{b\}, \phi, \phi\rangle)=\langle\{b\}, \phi, \phi\rangle$, $\ell(\langle\{c\}, \phi, \phi\rangle)=\ell(\langle\{d\}, \phi, \phi\rangle)=\langle\{d\}, \phi, \phi\rangle$. We observe that $\ell$ is a NC $\alpha$-open mapping, which is not NC-open mapping since $\langle\{a, b, c\}, \phi, \phi\rangle$ is NC-OS in $\mathcal{U}$, but $\ell(\langle\{a, b, c\}, \phi, \phi\rangle)=\langle\{a, b, d\}, \phi, \phi\rangle$ is not a NC-OS in $\mathcal{U}$.

Example 3.5: Let $\mathcal{U}=\{a, b, c, d\}$.

Then, $\tau_{U}=\left\{\phi_{N},\langle\{a\}, \phi, \phi\rangle,\langle\{b\}, \phi, \phi\rangle,\langle\{a, b\}, \phi, \phi\rangle,\langle\{a, b, c\}, \phi, \phi\rangle, \mathcal{U}_{N}\right\}$ is a NCTS. The class of all NC $\alpha$-OSs of $\mathcal{U}$ is: $\mathrm{NC} \alpha \mathrm{O}(\mathcal{U})=\tau_{u} \cup\{\langle\{a, b, d\}, \phi, \phi\rangle\}$. The class of all NCS $\alpha$-OSs of $\mathcal{U}$ is: $\mathrm{NCS} \alpha \mathrm{O}(\mathcal{U})=\mathrm{NC} \alpha \mathrm{O}(\mathcal{U}) \cup\{\langle\{a, c\}, \phi, \phi\rangle,\langle\{a, d\}, \phi, \phi\rangle,\langle\{b, c\}, \phi, \phi\rangle,\langle\{b, d\}, \phi, \phi\rangle,\langle\{a, c, d\}, \phi, \phi\rangle$, $\langle\{b, c, d\}, \phi, \phi\rangle\}$.

We define a mapping $\ell: \mathcal{U} \rightarrow \mathcal{U}$ by $\ell(\langle\{a\}, \phi, \phi\rangle)=\ell(\langle\{b\}, \phi, \phi\rangle)=\langle\{a\}, \phi, \phi\rangle, \ell(\langle\{c\}, \phi, \phi\rangle)=$ $\ell(\langle\{d\}, \phi, \phi\rangle)=\langle\{c\}, \phi, \phi\rangle$. It is easily seen that $\ell$ is a NCS $\alpha$-open mapping, but it is not NC-open mapping since $\langle\{a, b, c\}, \phi, \phi\rangle$ is NC-OS in $\mathcal{U}$, but $\ell(\langle\{a, b, c\}, \phi, \phi\rangle)=\langle\{a, c\}, \phi, \phi\rangle$ is not a NC-OS in $\mathcal{U}$. Then, $\ell$ is a NCS $\alpha$-open mapping, but it is not NC $\alpha$-open mapping.

Remark 3.6: The ideas of NC-open mapping and $\mathrm{NC}^{*}$-open mapping are self-regulating, as in the further examples below:

Example 3.7: Let $\mathcal{U}=\{a, b, c, d\}$.

Then, $\tau_{\mathcal{U}}=\left\{\phi_{N},\langle\{a\}, \phi, \phi\rangle,\langle\{b\}, \phi, \phi\rangle,\langle\{a, b\}, \phi, \phi\rangle,\langle\{a, b, c\}, \phi, \phi\rangle, \mathcal{U}_{N}\right\}$ is a NCTS. The class of all $\mathrm{NC} \alpha$-OSs of $\mathcal{U}$ is: $\mathrm{NC} \alpha \mathrm{O}(\mathcal{U})=\tau_{u} \cup\{\langle\{a, b, d\}, \phi, \phi\rangle\}$. The class of all NCS $\alpha$-OSs of $\mathcal{U}$ is: $\mathrm{NCS} \alpha \mathrm{O}(\mathcal{U})=\mathrm{NC} \alpha \mathrm{O}(\mathcal{U}) \cup\{\langle\{a, c\}, \phi, \phi\rangle,\langle\{a, d\}, \phi, \phi\rangle,\langle\{b, c\}, \phi, \phi\rangle,\langle\{b, d\}, \phi, \phi\rangle,\langle\{a, c, d\}, \phi, \phi\rangle$, $\langle\{b, c, d\}, \phi, \phi\rangle\}$.

We define a mapping $\ell: \mathcal{U} \rightarrow \mathcal{U}$ by $\ell(\langle\{a\}, \phi, \phi\rangle)=\ell(\langle\{b\}, \phi, \phi\rangle)=\langle\{a\}, \phi, \phi\rangle, \ell(\langle\{c\}, \phi, \phi\rangle)=$ $\langle\{b\}, \phi, \phi\rangle, \ell(\langle\{d\}, \phi, \phi\rangle)=\langle\{c\}, \phi, \phi\rangle$. It is easily seen that $\ell$ is a NC-open mapping, which is not $\mathrm{NC} \alpha^{*}$-open mapping since $\langle\{a, b, d\}, \phi, \phi\rangle \in \mathrm{NC} \alpha \mathrm{O}(\mathcal{U})$, but $\ell(\langle\{a, b, d\}, \phi, \phi\rangle)=\langle\{a, c\}, \phi, \phi\rangle \notin$ $\mathrm{NC} \alpha \mathrm{O}(\mathcal{U})$.

Example 3.8: In Example 3.4, it is easily seen that $\ell$ is a $\mathrm{NC}^{*}$-open mapping, but it is not NC-open since $\langle\{a, b, c\}, \phi, \phi\rangle \in \tau_{\mathcal{U}}$, but $\ell(\langle\{a, b, c\}, \phi, \phi\rangle)=\langle\{a, b, d\}, \phi, \phi\rangle \notin \tau_{\mathcal{U}}$.

\section{Proposition 3.9}

1) If $\ell:(U, \tau) \rightarrow(\mathcal{V}, \sigma)$ is a NC-open, NC-continuous mapping, then $\ell$ is a $\mathrm{NC}^{*}$-open mapping.

2) $\quad \ell:(\mathcal{U}, \tau) \rightarrow(\mathcal{V}, \sigma)$ is a $\mathrm{NC}^{*}$-open mapping iff $\ell:(\mathcal{U}, \mathrm{NC \alpha O}(\mathcal{U})) \rightarrow(\mathcal{V}, \mathrm{NC} \alpha \mathrm{O}(\mathcal{V}))$ is a NCopen. 


\section{Proof}

1) Let $\ell:(\mathcal{U}, \tau) \rightarrow(\mathcal{V}, \sigma)$ be a NC-open, NC-continuous mapping. To prove that $\ell$ is a NC $\alpha^{*}$ open mapping, let $\mathcal{M} \in \mathrm{NC} \alpha \mathrm{O}(\mathcal{U})$, then for some $\mathrm{NC}-\mathrm{OS} \mathcal{F}$, such that $\mathcal{F} \subseteq \mathcal{M} \subseteq \operatorname{NCint}(\operatorname{NCcl}(\mathcal{F}))$ (by Proposition 2.5). Hence, $\ell(\mathcal{F}) \subseteq \ell(\mathcal{M}) \subseteq \ell(N \operatorname{Cint}(N \operatorname{Ccl}(\mathcal{F})))$, but $\ell(N \operatorname{Cint}(\operatorname{NCcl}(\mathcal{F}))) \subseteq$ $N \operatorname{Cint}(\ell(N \operatorname{Ccl}(\mathcal{F})))$ (since $\ell$ is a NC-open mapping).

Then, $\ell(\mathcal{F}) \subseteq \ell(\mathcal{M}) \subseteq \ell(N \operatorname{Cint}(N \operatorname{Ccl}(\mathcal{F}))))) \subseteq N \operatorname{Cint}(\ell(N \operatorname{Ccl}(\mathcal{F})))$.

But, $N \operatorname{Cint}(\ell(N \operatorname{Ccl}(\mathcal{F}))) \subseteq N \operatorname{Cint}(N \operatorname{Ccl}(\ell(\mathcal{F}))$ ) (since $\ell$ is a NC-continuous mapping). Therefore, we get $\ell(\mathcal{F}) \subseteq \ell(\mathcal{M}) \subseteq N \operatorname{Cint}(\operatorname{NCCl}(\ell(\mathcal{F}))$ ). However, $\ell(\mathcal{F})$ is a NC-OS in $\mathcal{V}$ (since $\ell$ is a NC-open mapping). Hence, $\ell(\mathcal{M}) \in \mathrm{NC} \alpha \mathrm{O}(\mathcal{V})$ (by Proposition 2.5). Thus, it is a $\mathrm{NC}^{*}$-open mapping.

2) The proof of a part (2) is easily reached.

Proposition 3.10: Every $\mathrm{NC}^{*}$-open mapping is a $\mathrm{NC} \alpha$-open and $\mathrm{NCS} \alpha$-open, but the reverse is not valid in general.

Proof: Let $\ell:(U, \tau) \rightarrow(\mathcal{V}, \sigma)$ be a NC $\alpha^{*}$-open mapping and $\mathcal{M}$ be NC-OS in $\mathcal{U}$. Then, we have that $\mathcal{M}$ is considered a $\mathrm{NC} \alpha$-OS in $\mathcal{U}$ [from Proposition 2.5]. Because $\ell$ is a $\mathrm{NC} \alpha *$-open, then $\ell(\mathcal{M})$ is considered a NC $\alpha$-OS in $\mathcal{V}$. Therefore, $\ell$ is a NC $\alpha$-open. Also, $\ell$ is a NCS $\alpha$-open.

Example 3.11: In Example 3.7, it is easily seen that $\ell$ is a NC $\alpha$-open mapping and NCS $\alpha$-open mapping, but not $\mathrm{NC}^{*}$-open.

Remark 3.12: The ideas of NC-open mapping and $\mathrm{NCS} \alpha{ }^{*}$-open mapping are independent, as explained in the examples below.

Example 3.13: Let $\mathcal{U}=\{a, b, c\}$. Then, $\tau=\left\{\phi_{N},\langle\{a\}, \phi, \phi\rangle, \mathcal{U}_{N}\right\}$ is a NCTS. The class of all NC $\alpha-$ OSs (NCS $\alpha$-OSs) of $\mathcal{U}$ is: $\mathrm{NC} \alpha \mathrm{O}(\mathcal{U})=\operatorname{NCS} \alpha \mathrm{O}(\mathcal{U})=\tau \cup\{\langle\{a, b\}, \phi, \phi\rangle,\langle\{a, c\}, \phi, \phi\rangle\}$. Let $\mathcal{V}=$ $\{p, q, r, s\}$. Then, $\sigma=\left\{\phi_{N},\langle\{p\}, \phi, \phi\rangle,\langle\{q, r\}, \phi, \phi\rangle,\langle\{p, q, r\}, \phi, \phi\rangle, \mathcal{V}_{N}\right\}$ is a NCTS. The class of all $\mathrm{NC} \alpha$-OSs of $\mathcal{V}$ is: $\mathrm{NC} \alpha \mathrm{O}(\mathcal{V})=\sigma$. The class of all NCS $\alpha$-OSs of $\mathcal{V}$ is: $\mathrm{NCS} \alpha \mathrm{O}(\mathcal{V})=\mathrm{NC} \alpha \mathrm{O}(\mathcal{V}) \cup\{\langle\{q, r, s\}, \phi, \phi\rangle\}$.

We define a mapping $\ell: \mathcal{U} \rightarrow \mathcal{V}$ by $\ell(\langle\{a\}, \phi, \phi\rangle)=\langle\{p\}, \phi, \phi\rangle, \ell(\langle\{b\}, \phi, \phi\rangle)=\langle\{q\}, \phi, \phi\rangle$, $\ell(\langle\{c\}, \phi, \phi\rangle)=\langle\{r\}, \phi, \phi\rangle$. It is easily seen that $\ell$ is a NC-open mapping, but it is not NCSa*-open mapping, since $\langle\{a, b\}, \phi, \phi\rangle \in \mathrm{NCS} \alpha \mathrm{O}(\mathcal{U})$, but $\ell(\langle\{a, b\}, \phi, \phi\rangle)=\langle\{p, q\}, \phi, \phi\rangle \notin \operatorname{NCS} \alpha \mathrm{O}(\mathcal{V})$.

Example 3.14: Let $\mathcal{U}=\{a, b, c\}$. Then, $\tau_{u}=\left\{\phi_{N},\langle\{a\}, \phi, \phi\rangle,\langle\{b\}, \phi, \phi\rangle,\langle\{a, b\}, \phi, \phi\rangle, \mathcal{U}_{N}\right\}$ is a NCTS. The family of all NC $\alpha$-OSs of $\mathcal{U}$ is: $\operatorname{NC} \alpha \mathrm{O}(\mathcal{U})=\tau_{\mathcal{U}}$. The family of all NCS $\alpha$-OSs of $\mathcal{U}$ is: $\mathrm{NC} \alpha \mathrm{O}(\mathcal{U})=\mathrm{NC} \alpha \mathrm{O}(\mathcal{U}) \cup\{\langle\{a, c\}, \phi, \phi\rangle,\langle\{b, c\}, \phi, \phi\rangle\}$.

We define a mapping $\ell: \mathcal{U} \rightarrow \mathcal{U}$ by $\ell(\langle\{a\}, \phi, \phi\rangle)=\ell(\langle\{b\}, \phi, \phi\rangle)=\langle\{a\}, \phi, \phi\rangle, \ell(\langle\{c\}, \phi, \phi\rangle)=$ $\langle\{c\}, \phi, \phi\rangle$. It is easily observed that $\ell$ is a NCS $\alpha^{*}$-open mapping, but it is not NC-open mapping since $\mathcal{U}_{N} \in \tau_{u}$, but $\ell\left(\mathcal{U}_{N}\right)=\langle\{a, c\}, \phi, \phi\rangle \notin \tau_{u}$.

Proposition 3.15: A mapping $\ell:(\mathcal{U}, \tau) \rightarrow(\mathcal{V}, \sigma)$ is a $\mathrm{NCS}^{*}$-open iff $\ell:(\mathcal{U}, \mathrm{NCS} \alpha O(\mathcal{U})) \rightarrow$ $(\mathcal{V}, \mathrm{NCS} \alpha \mathrm{O}(\mathcal{V}))$ is a NC-open mapping.

Proof: Obvious.

Remark 3.16: The ideas of $\mathrm{NC}^{*}$-open mapping and $\mathrm{NCS}^{*}{ }^{*}$-open mapping are independent as the further examples demonstrate:

Example 3.17: In Example 3.14, it is easily seen that $\ell$ is a NCS $\alpha^{*}$-open mapping but it is not $\mathrm{NC}^{*}{ }_{-}$ open since $U_{N} \in \mathrm{NC} \alpha \mathrm{O}(\mathcal{U})$, but $\ell\left(U_{N}\right)=\langle\{a, c\}, \phi, \phi\rangle \notin \mathrm{NC} \alpha \mathrm{O}(\mathcal{U})$.

Example 3.18: Let $\mathcal{U}=\{a, b, c, d\}$. Then $\tau=\left\{\phi_{N},\langle\{a\}, \phi, \phi\rangle,\langle\{b, d\}, \phi, \phi\rangle,\langle\{a, b, d\}, \phi, \phi\rangle, \mathcal{U}_{N}\right\}$ is a NCTS. Let $\mathcal{V}=\{p, q, r, s\}$. Then $\sigma=\left\{\phi_{N},\langle\{p\}, \phi, \phi\rangle,\langle\{q, s\}, \phi, \phi\rangle,\langle\{p, q, s\}, \phi, \phi\rangle, \mathcal{V}_{N}\right\}$ is a NCTS.

Define a mapping $\ell: \mathcal{U} \rightarrow \mathcal{V}$ by $\ell(\langle\{a\}, \phi, \phi\rangle)=\langle\{p\}, \phi, \phi\rangle, \ell(\langle\{b\}, \phi, \phi\rangle)=\ell(\langle\{c\}, \phi, \phi\rangle)=$ $\langle\{q\}, \phi, \phi\rangle, \ell(\langle\{d\}, \phi, \phi\rangle)=\langle\{s\}, \phi, \phi\rangle$. It is easily seen that $\ell$ is a $\mathrm{NC}^{*}$-open mapping, but it is not $\mathrm{NCS} \alpha *$-open mapping.

Theorem 3.19: If a mapping $\ell:(U, \tau) \rightarrow(\mathcal{V}, \sigma)$ is $\mathrm{NC}^{*}$-open and NC-continuous, then it is NCS $\alpha^{*}$ open.

Proof: Let $\ell:(\mathcal{U}, \tau) \rightarrow(\mathcal{V}, \sigma)$ be a NC $\alpha^{*}$-open and NC-continuous mapping. Let $\mathcal{M}$ be a NCS $\alpha$-OS in $\mathcal{U}$. Then, we have for some NCa-OS, say $\mathcal{S}$, such that $\mathcal{S} \subseteq \mathcal{M} \subseteq \operatorname{NCcl}(\mathcal{S})$. Therefore, $\ell(\mathcal{S}) \subseteq$ $\ell(\mathcal{M}) \subseteq \ell(N C c l(\mathcal{S})) \subseteq N C \operatorname{Nol}(\ell(\mathcal{S})$ ) (since $\ell$ is a NC-continuous), but $\ell(\mathcal{S}) \in \mathrm{NC} \alpha \mathrm{O}(\mathcal{U})$ (since $\ell$ is a $\mathrm{NC} \alpha *$-open mapping). Hence, $\ell(\mathcal{S}) \subseteq \ell(\mathcal{M}) \subseteq N \operatorname{Ccl}(\ell(\mathcal{S})$ ). Thus, $\ell(\mathcal{M}) \in \mathrm{NCS} \alpha \mathrm{O}(\mathcal{U})$. Therefore, $\ell$ is a $\mathrm{NCS} \alpha *$-open. 
Theorem 3.20: Let $\ell_{1}:(\mathcal{U}, \tau) \rightarrow(\mathcal{V}, \sigma)$ and $\ell_{2}:(\mathcal{V}, \sigma) \rightarrow(\mathcal{W}, \rho)$ be two mappings, then:

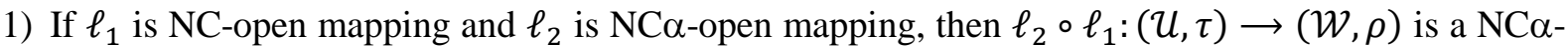
open mapping.

2) If $\ell_{1}$ is $\mathrm{NC} \alpha$-open mapping and $\ell_{2}$ is $\mathrm{NC}^{*}$-open mapping, then $\ell_{2} \circ \ell_{1}:(\mathcal{U}, \tau) \rightarrow(\mathcal{W}, \rho)$ is a $\mathrm{NC} \alpha$-open mapping.

3) If $\ell_{1}$ and $\ell_{2}$ are $\mathrm{NC}^{*}$-open mappings, then $\ell_{2} \circ \ell_{1}:(\mathcal{U}, \tau) \rightarrow(\mathcal{W}, \rho)$ is a $\mathrm{NC} \alpha^{*}$-open mapping.

4) If $\ell_{1}$ and $\ell_{2}$ are NCS $\alpha^{*}$-open mappings, then $\ell_{2} \circ \ell_{1}:(U, \tau) \rightarrow(\mathcal{W}, \rho)$ is a NCS $\alpha^{*}$-open mapping.

5) If $\ell_{1}$ and $\ell_{2}$ are $\mathrm{NC}^{* *}$-open mappings, then $\ell_{2} \circ \ell_{1}:(\mathcal{U}, \tau) \rightarrow(\mathcal{W}, \rho)$ is a $\mathrm{NC}^{* *}$-open mapping.

6) If $\ell_{1}$ and $\ell_{2}$ are $\mathrm{NCS}^{* *}{ }^{*}$-open mappings, then $\ell_{2} \circ \ell_{1}:(\mathcal{U}, \tau) \rightarrow(\mathcal{W}, \rho)$ is a NCS$\alpha^{* *}$-open mapping.

7) If $\ell_{1}$ is $\mathrm{NC}^{* * *}$-open mapping and $\ell_{2}$ is $\mathrm{NC}^{*}$-open mapping, then $\ell_{2} \circ \ell_{1}:(\mathcal{U}, \tau) \rightarrow(\mathcal{W}, \rho)$ is a $\mathrm{NC} \alpha *$-open mapping.

8) If $\ell_{1}$ is $\mathrm{NC} \alpha$-open mapping and $\ell_{2}$ is $\mathrm{NC}^{* *}$-open mapping, then $\ell_{2} \circ \ell_{1}:(\mathcal{U}, \tau) \rightarrow(\mathcal{W}, \rho)$ is a NC-open mapping.

9) If $\ell_{1}$ is $\mathrm{NC} \alpha^{* *}$-open mapping and $\ell_{2}$ is $\mathrm{NC} \alpha$-open mapping, then $\ell_{2} \circ \ell_{1}:(\mathcal{U}, \tau) \rightarrow(\mathcal{W}, \rho)$ is a $\mathrm{NC} \alpha^{*}$-open mapping.

10) If $\ell_{1}$ is $\mathrm{NC}^{* *}$-open mapping and $\ell_{2}$ is NC-open mapping, then $\ell_{2} \circ \ell_{1}:(\mathcal{U}, \tau) \rightarrow(\mathcal{W}, \rho)$ is a $\mathrm{NC} \alpha^{* *}$-open mapping.

\section{Proof}

1) Let a NC-OS $\mathcal{M}$ be in $\mathcal{U}$. Since $\ell_{1}$ is a NC-open mapping, then $\ell_{1}(\mathcal{M})$ is considered as a NC-OS in $\mathcal{V}$. Because $\ell_{2}$ is a NC $\alpha$-open mapping, $\ell_{2} \circ \ell_{1}(\mathcal{M})=\ell_{2}\left(\ell_{1}(\mathcal{M})\right)$ is considered as a NC $\alpha$-OS in $\mathcal{W}$. Thus, $\ell_{2} \circ \ell_{1}:(\mathcal{U}, \tau) \rightarrow(\mathcal{W}, \rho)$ is a $\mathrm{NC} \alpha$-open mapping.

2) Let a NC-OS $\mathcal{M}$ be in $U$. Since $\ell_{1}$ is a NC $\alpha$-open mapping, then $\ell_{1}(\mathcal{M})$ is considered as a NC $\alpha$ OS in $\mathcal{V}$. Because $\ell_{2}$ is a $\mathrm{NC}^{*}$-open mapping, $\ell_{2} \circ \ell_{1}(\mathcal{M})=\ell_{2}\left(\ell_{1}(\mathcal{M})\right)$ is considered as a NC $\alpha$ OS in $\mathcal{W}$. Thus, $\ell_{2} \circ \ell_{1}:(\mathcal{U}, \tau) \rightarrow(\mathcal{W}, \rho)$ is a NC $\alpha$-open mapping.

3) Let a $\mathrm{NC} \alpha$-OS $\mathcal{M}$ be in $\mathcal{U}$. Since $\ell_{1}$ is a $\mathrm{NC}^{*}$-open mapping, then $\ell_{1}(\mathcal{M})$ is considered as a NC $\alpha$-OS in $\mathcal{V}$. Because $\ell_{2}$ is a NC $\alpha^{*}$-open mapping, $\ell_{2} \circ \ell_{1}(\mathcal{M})=\ell_{2}\left(\ell_{1}(\mathcal{M})\right)$ is considered as a $\mathrm{NC} \alpha$-OS in $\mathcal{W}$. Thus, $\ell_{2} \circ \ell_{1}:(\mathcal{U}, \tau) \rightarrow(\mathcal{W}, \rho)$ is a $\mathrm{NC}^{*}$-open mapping.

4) Let a NCS $\alpha$-OS $\mathcal{M}$ be in $\mathcal{U}$. Since $\ell_{1}$ is a NCS $\alpha^{*}$-open mapping, then $\ell_{1}(\mathcal{M})$ is considered as a NCS $\alpha$-OS in $\mathcal{V}$. Because $\ell_{2}$ is a NCS $\alpha^{*}$-open mapping, $\ell_{2} \circ \ell_{1}(\mathcal{M})=\ell_{2}\left(\ell_{1}(\mathcal{M})\right)$ is considered as a NCS $\alpha$-OS in $\mathcal{W}$. Thus, $\ell_{2} \circ \ell_{1}:(\mathcal{U}, \tau) \rightarrow(\mathcal{W}, \rho)$ is a NCS $\alpha *$-open mapping.

5) Let a $\mathrm{NC} \alpha$-OS $\mathcal{M}$ be in $\mathcal{U}$. Since $\ell_{1}$ is a $\mathrm{NC}^{* *}$-open mapping, then $\ell_{1}(\mathcal{M})$ is considered as a NC-OS in $\mathcal{V}$. Because any NC-OS is NC $\alpha$-OS, $\ell_{1}(\mathcal{M})$ is considered as a NC $\alpha$-OS in $\mathcal{V}$. Meanwhile $\ell_{2}$ is a NCa**-open mapping, then $\ell_{2} \circ \ell_{1}(\mathcal{M})=\ell_{2}\left(\ell_{1}(\mathcal{M})\right)$ is considered as a NC-OS in $\mathcal{W}$. Thus, $\ell_{2} \circ \ell_{1}:(\mathcal{U}, \tau) \rightarrow(\mathcal{W}, \rho)$ is a $\mathrm{NC}^{* *}{ }_{\text {-open mapping. }}$

6) Let a NCS $\alpha$-OS $\mathcal{M}$ be in $\mathcal{U}$. Since $\ell_{1}$ is a NCS $\alpha^{* *}$-open mapping, then $\ell_{1}(\mathcal{M})$ is considered as a NC-OS in $\mathcal{V}$. Because any NC-OS is NCS $\alpha-O S, \ell_{1}(\mathcal{M})$ is considered as a NCS $\alpha$-OS in $\mathcal{V}$. Meanwhile, $\ell_{2}$ is a NCS $\alpha^{* *}$-open mapping and $\ell_{2} \circ \ell_{1}(\mathcal{M})=\ell_{2}\left(\ell_{1}(\mathcal{M})\right)$ is considered as a NC-OS in $\mathcal{W}$. Thus, $\ell_{2} \circ \ell_{1}:(\mathcal{U}, \tau) \rightarrow(\mathcal{W}, \rho)$ is a $\mathrm{NCS}^{* *}$-open mapping.

7) Let a NC $\alpha$-OS $\mathcal{M}$ be in $\mathcal{U}$. Since $\ell_{1}$ is a $\mathrm{NC}^{* *}$-open mapping, $\ell_{1}(\mathcal{M})$ is considered as a NCOS in $\mathcal{V}$. Because any NC-OS is NC $\alpha$-OS, $\ell_{1}(\mathcal{M})$ is considered as a NC $\alpha$-OS in $\mathcal{V}$. Meanwhile $\ell_{2}$ is a $\mathrm{NC}^{*}$-open mapping and $\ell_{2} \circ \ell_{1}(\mathcal{M})=\ell_{2}\left(\ell_{1}(\mathcal{M})\right)$ is considered as a $\mathrm{NC} \alpha$-OS in $\mathcal{W}$. Thus, $\ell_{2} \circ \ell_{1}:(\mathcal{U}, \tau) \rightarrow(\mathcal{W}, \rho)$ is a $\mathrm{NC}^{*}$-open mapping.

8) Let a NC-OS $\mathcal{M}$ be in $\mathcal{U}$. Since $\ell_{1}$ is a NC $\alpha$-open mapping, then $\ell_{1}(\mathcal{M})$ is considered as a NC $\alpha$ OS in $\mathcal{V}$. Because $\ell_{2}$ is a NC $\alpha^{* *}$-open mapping, $\ell_{2} \circ \ell_{1}(\mathcal{M})=\ell_{2}\left(\ell_{1}(\mathcal{M})\right)$ is considered as a NC-OS in $\mathcal{W}$. Thus, $\ell_{2} \circ \ell_{1}:(\mathcal{U}, \tau) \rightarrow(\mathcal{W}, \rho)$ is a NC-open mapping.

9) Let a NC $\alpha$-OS $\mathcal{M}$ be in $\mathcal{U}$. Since $\ell_{1}$ is a $\mathrm{NC}^{* *}$-open mapping, then $\ell_{1}(\mathcal{M})$ is considered as a NC-OS in $\mathcal{V}$. Because $\ell_{2}$ is a NC $\alpha$-open mapping, $\ell_{2} \circ \ell_{1}(\mathcal{M})=\ell_{2}\left(\ell_{1}(\mathcal{M})\right)$ is considered as a NC $\alpha$ OS in $\mathcal{W}$. Thus, $\ell_{2} \circ \ell_{1}:(\mathcal{U}, \tau) \rightarrow(\mathcal{W}, \rho)$ is a $\mathrm{NC}^{*}$-open mapping. 
10) Let a $\mathrm{NC} \alpha$-OS $\mathcal{M}$ be in $\mathcal{U}$. Since $\ell_{1}$ is a $\mathrm{NC}^{* *}$-open mapping, then $\ell_{1}(\mathcal{M})$ is considered as a NC-OS in $\mathcal{V}$. Because $\ell_{2}$ is a NC-open mapping, $\ell_{2} \circ \ell_{1}(\mathcal{M})=\ell_{2}\left(\ell_{1}(\mathcal{M})\right)$ is considered as a NC-OS in $\mathcal{W}$. Thus, $\ell_{2} \circ \ell_{1}:(\mathcal{U}, \tau) \rightarrow(\mathcal{W}, \rho)$ is a $\mathrm{NC}^{* *}$-open mapping.

Remark 3.21: The illustration demonstrated in Figure 1 explains the relationships between weakly NC-open mappings.

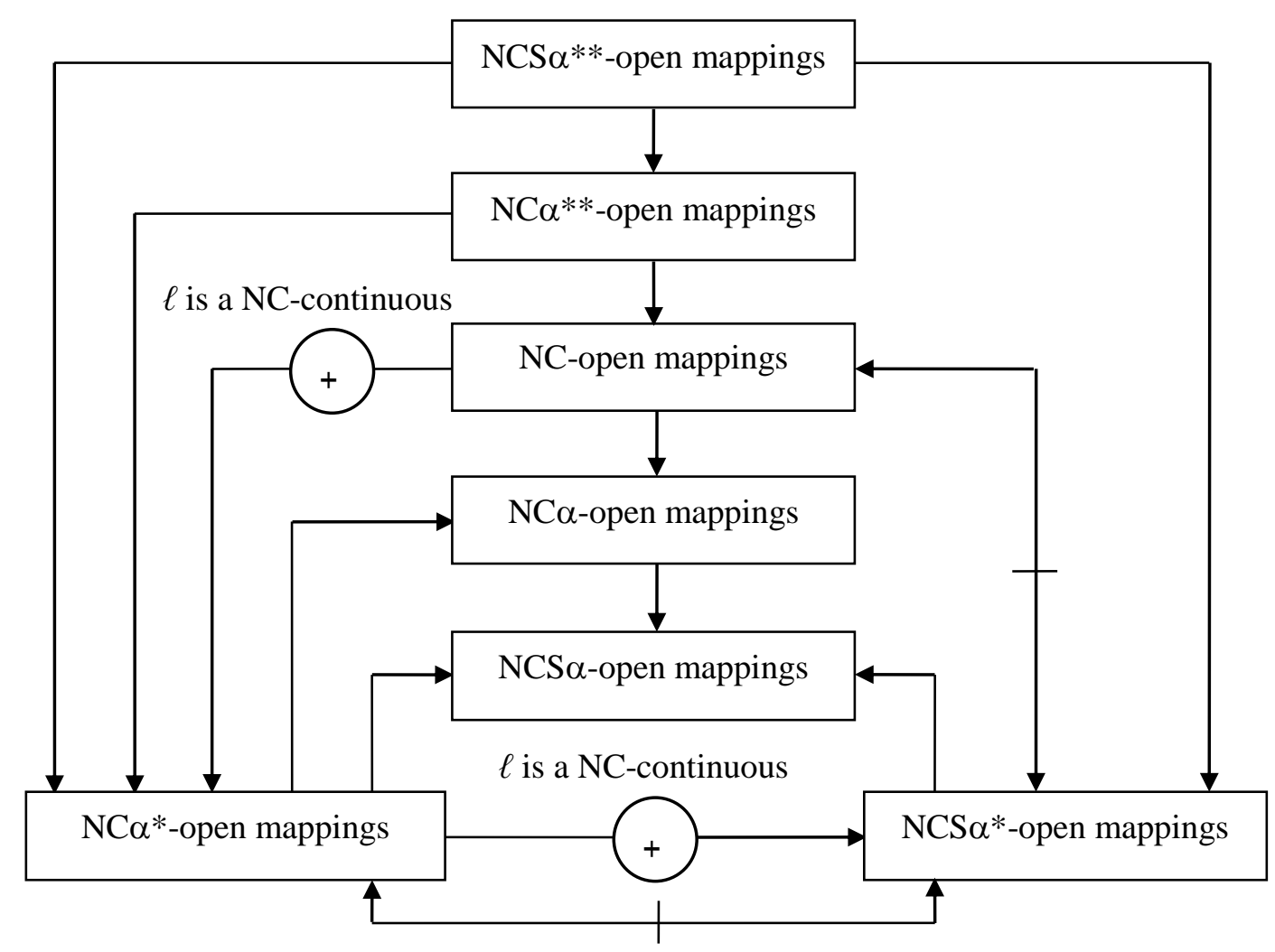

Figure 1- The relationships between weakly NC-open mappings.

\section{Conclusions}

We developed the thoughts of $\mathrm{NC} \alpha$-open and NCS $\alpha$-open sets to describe some fresh types of weakly neutrosophic crisp open mappings, such as $\mathrm{NC} \alpha$-open, $\mathrm{NC} \alpha^{*}$-open, $\mathrm{NC} \alpha^{* *}$-open, $\mathrm{NCS} \alpha-$ open, $\mathrm{NCS}^{*}$-open, and NCS $\alpha^{* *}$-open mappings. The NC $\alpha$-closed and NCS $\alpha$-closed sets can be manipulated to obtain some innovative kinds of weakly neutrosophic crisp closed mappings.

\section{References}

1. Salama, A. A., Smarandache, F. and Kroumov, V. 2014. Neutrosophic crisp sets and neutrosophic crisp topological spaces, Neutrosophic Sets and Systems, 2: 25-30.

2. Abdel-Basset, M., Mohamed, R., Zaied, A.E.N.H. and Smarandache, F. 2019. A Hybrid Plithogenic Decision-Making Approach with Quality Function Deployment for Selecting Supply Chain Sustainability Metrics, Symmetry, 11(7): 903.

3. Abdel-Basset, M., Nabeeh, N. A., El-Ghareeb, H. A. And Aboelfetouh, A. 2019. Utilising neutrosophic theory to solve transition difficulties of IoT-based enterprises, Enterprise Information Systems, pp:1-21.

4. Abdel-Baset, M., Chang, V. and Gamal, A. 2019. Evaluation of the green supply chain management practices: A novel neutrosophic approach, Computers in Industry, 108:210-220.

5. Abdel-Basset, M., Saleh, M., Gamal, A. and Smarandache, F. 2019. An approach of TOPSIS technique for developing supplier selection with group decision making under type-2 neutrosophic number, Applied Soft Computing, 77:438-452. 
6. Abdel-Baset, M., Chang, V., Gamal, A. and Smarandache, F. 2019. An integrated neutrosophic ANP and VIKOR method for achieving sustainable supplier selection: A case study in importing field, Computers in Industry, 106:94-110.

7. Abdel-Basset, M., Manogaran, G., Gamal, A.and Smarandache, F. 2019. A group decision making framework based on neutrosophic TOPSIS approach for smart medical device selection, Journal of medical systems, $\mathbf{4 3}(2): 38$.

8. Al-Hamido, R. K., Imran, Q. H., Alghurabi, K. A. and Gharibah, T. 2018. On neutrosophic crisp semi- $\alpha$-closed sets, Neutrosophic Sets and Systems, 21:28-35.

9. Banupriya, V. and Chandrasekar, S. 2019. Neutrosophic ags Continuity and Neutrosophic ags Irresolute Maps, Neutrosophic Sets and Systems, 28:162-170.

10. Dhavaseelan, R., Narmada Devi, R., Jafari, S. and Imran, Q. H. 2019. Neutrosophic $\alpha^{\mathrm{m}}$ continuity, Neutrosophic Sets and Systems, 27:171-179.

11. Maheswari, C. and Chandrasekar, S. 2019. Neutrosophic gb-closed Sets and Neutrosophic gbContinuity, Neutrosophic Sets and Systems, 29:89-100.

12. Salama, A. A. 2015. Basic structure of some classes of neutrosophic crisp nearly open sets \& possible application to GIS topology, Neutrosophic Sets and Systems, 7:18-22.

13. Al-Omeri, W. 2016. Neutrosophic crisp sets via neutrosophic crisp topological spaces, Neutrosophic Sets and Systems, 13:96-104.

14. Salama, A. A., Hanafy, I. M., Elghawalby, H. and Dabash, M. S. 2016. Some GIS Topological Concepts via Neutrosophic Crisp Set Theory, New Trends in Neutrosophic Theory and Applications. 